\title{
Wavelength-independent laser beam shaping
}

\author{
Mapule P. Degama ${ }^{\mathrm{a}, \mathrm{b}}$, Andrew Forbes*a,b,c \\ ${ }^{a}$ CSIR National Laser Centre, PO Box 395, Pretoria 0001, South Africa; \\ ${ }^{\mathrm{b}}$ School of Physics, University of Kwazulu-Natal, Private Bag X54001, Durban 4000, South Africa \\ ${ }^{c}$ Department of Physics, Stellenbosch University, Private Bag X1, Matieland 7602, South Africa
}

\begin{abstract}
This paper presents a beam shaping device namely, a Diffractive Optical Element (DOE), which is used to change a beam having a Gaussian intensity profile into a beam with a uniform intensity profile. The DOE used in this work was fabricated from $\mathrm{ZnSe}$ and its performance was evaluated using a cw $\mathrm{CO}_{2}$ laser. In most cases such elements are effective only at a specific design wavelength. However, in this paper we report on the design conditions which allow for wavelength independent elements. It was found that the DOE was able to successfully transform a Gaussian beam into a flattop beam for four different wavelengths in the range $9.2 \mu \mathrm{m}$ to $10.6 \mu \mathrm{m}$. We also present experimental results on misalignment effects and it was found that small radial offsets of the incident beam on the DOE had a significant disruptive effect on the flattop beam profile.
\end{abstract}

Keywords: Diffractive Optical Element (DOE), wavelength independent elements, laser beam shaping, flattop beam

\section{INTRODUCTION}

Laser beam shaping allows for the intensity distribution of a laser beam to be modified from that emitted from the laser resonator. This process has certain advantages in some industrial and research applications such as laser materials processing $^{1}$, optical data storage ${ }^{2}$ and laser therapy ${ }^{3}$. Many of these applications require a uniform illumination but typical laser sources have a Gaussian output, hence the need for laser beam shaping. Numerous optical systems ${ }^{4,5}$ and various design methods ${ }^{6,7}$ have been employed to address the problem of obtaining an optical element that converts a Gaussian intensity profile into a uniform intensity profile. Our approach to laser beam shaping consists of a diffractive optical element (DOE) as a phase element in conjunction with a Fourier transform lens. In this method a Gaussian beam is passed through a DOE, converting it into a flattop beam. A transforming lens placed directly behind the DOE, takes the Fourier transform of the incident Gaussian beam and produces a flattop intensity profile at the focal plane of the lens.

Fresnel diffraction theory is used to design the DOE which would transform a Gaussian beam into a flattop beam. The design parameters were then used to fabricate a $\mathrm{ZnSe}$ DOE. The performance of the DOE was then tested in beam shaping experiments using a continuous wave $(\mathrm{cw}) \mathrm{CO}_{2}$ laser. In section 2, we present the solution to the problem of determining the phase function of the DOE, while section 3 reports on the experimental investigations of the DOE laser beam shaping characteristics. Usually, such elements are effective only at a specific design wavelength ${ }^{8}$. We will concentrate on the design conditions that allow for wavelength independent elements. Experimental results on the effect of a radial offset of the initial beam on the DOE will be discussed, as well as the effects of using designed and nondesigned wavelengths in the $\mathrm{CO}_{2}$ laser tuneability range. The conclusions of the work are presented in section 4 .

\section{PHASE FUNCTION}

The solution for determining the phase function of the DOE is based on the Fresnel diffraction theory where, in one dimension, the approximated Fresnel diffraction integral takes the form ${ }^{9}$,

$$
g \boldsymbol{\emptyset}^{\prime}=\frac{1}{2 \pi} \int_{-\infty}^{\infty} f \boldsymbol{\varphi}_{-} \exp \boldsymbol{k} \phi \boldsymbol{d} \phi,
$$


where $g \boldsymbol{\zeta}^{\prime}$ - is the output field at the focal plane of the lens, $f \boldsymbol{\varphi}_{-}^{-}$is the input field, $\phi \boldsymbol{\varphi}_{-}^{-}$is the phase function of the DOE and $\rho$ is the transverse coordinate of the DOE. The solution to this problem depends on the parameter $\beta$, a dimensionless measure of how well the beam will be flattened and $\beta$ is defined as

$$
\beta=\frac{2 \pi \omega_{0} R_{0}}{f \lambda},
$$

where $\lambda$ is the wavelength of the beam, $\omega_{0}$ is the input beam radius, $R_{0}$ is the output beam radius and $f$ is the focal length of the transforming lens. It has been shown that the stationary phase solution improves as the increasing parameter $\beta$ increases ${ }^{10}$ illustrating that it is a key contribution to the beam shaping problem. The optical element is then designed to realize $\beta \phi \varphi_{\text {; }}$.

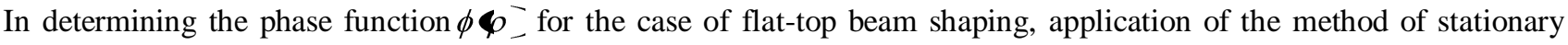
phase is used, where the stationary phase evaluation of the integral given by equation 1, leads to a second-order partial differential equation which solved results in

$$
g \varphi^{\prime}=\frac{1}{\sqrt{2 \pi}} \frac{f \varphi_{-}^{-}}{\sqrt{\beta \phi^{\prime \prime} \varphi^{-}}} .
$$

The phase function $\phi \varphi_{-}^{-}$, need to be solved so that the intensity, $g \varphi^{\prime}$, at the focal plane of the lens is approximately equal to some specified function $G \boldsymbol{\varphi}^{\prime}$, times a scale factor $\mu$. This is satisfied provided that

$$
\left|g \boldsymbol{\varphi}^{\prime}\right|^{2}=\mu G \boldsymbol{\phi}^{\prime} / \beta
$$

where $g \boldsymbol{6}^{\prime}$, is given by equation 1 and $\mu$ is chosen so that

$$
\mu \beta \int_{-\infty}^{\infty} G \varphi^{\prime} d \rho^{\prime}=\frac{1}{2 \pi} \int_{-\infty}^{\infty} \mid f \varphi I^{2} d \rho .
$$

In determining the solution for turning a Gaussian beam into a flattop beam, the incident field is given by the Gaussian intensity distribution as

$$
f \varphi_{-}^{-}=\exp \left(\frac{-\rho^{2}}{2}\right)
$$

and the output field is given by a constant intensity distribution:

$$
G \boldsymbol{\varphi}^{\prime}\left\{\begin{array}{l}
1, \text { for }\left|\rho^{\prime}\right|<\frac{\sqrt{\pi}}{2} \\
0, \text { for }\left|\rho^{\prime}\right|>\frac{\sqrt{\pi}}{2}
\end{array}\right. \text {. }
$$

Then equation 3 becomes

$$
f^{2} \varphi^{\prime}=2 \pi \beta \phi^{\prime \prime} \varphi \mu^{\prime}
$$

In order to solve this, the constant $\mu$ must be chosen such that equation 5 is satisfied. Hence, the scaling of the function $G \boldsymbol{\emptyset}^{\prime}$, is chosen so that the constant $\mu$ is equal to $1 / 2 \pi \beta$, yielding

$$
\begin{aligned}
\phi^{\prime \prime} \boldsymbol{\varphi}_{-}^{-} & =f^{2} \boldsymbol{\varphi}_{-} \\
& =\exp \leftarrow \rho^{2} \doteq
\end{aligned}
$$

By taking the second-order integration of both sides of equation 9 , the following result is obtained 


$$
\phi \boldsymbol{\varphi}_{-}^{-}=\rho \frac{\sqrt{\pi}}{2} \operatorname{erf} \boldsymbol{\varphi}_{-}+\frac{1}{2} \exp \leftarrow \rho^{2}=-\frac{1}{2} .
$$

Equation 10 is the solution for the phase function of the DOE in one-dimension, used to convert a Gaussian beam into a flattop beam ${ }^{10}$. Due to the absolute value sign in equation 4 , the negative of the solution in equation 10 is also a solution to the beam shaping problem. This result is important in some beam shaping application. The cross-section of the DOE is given in figure1.

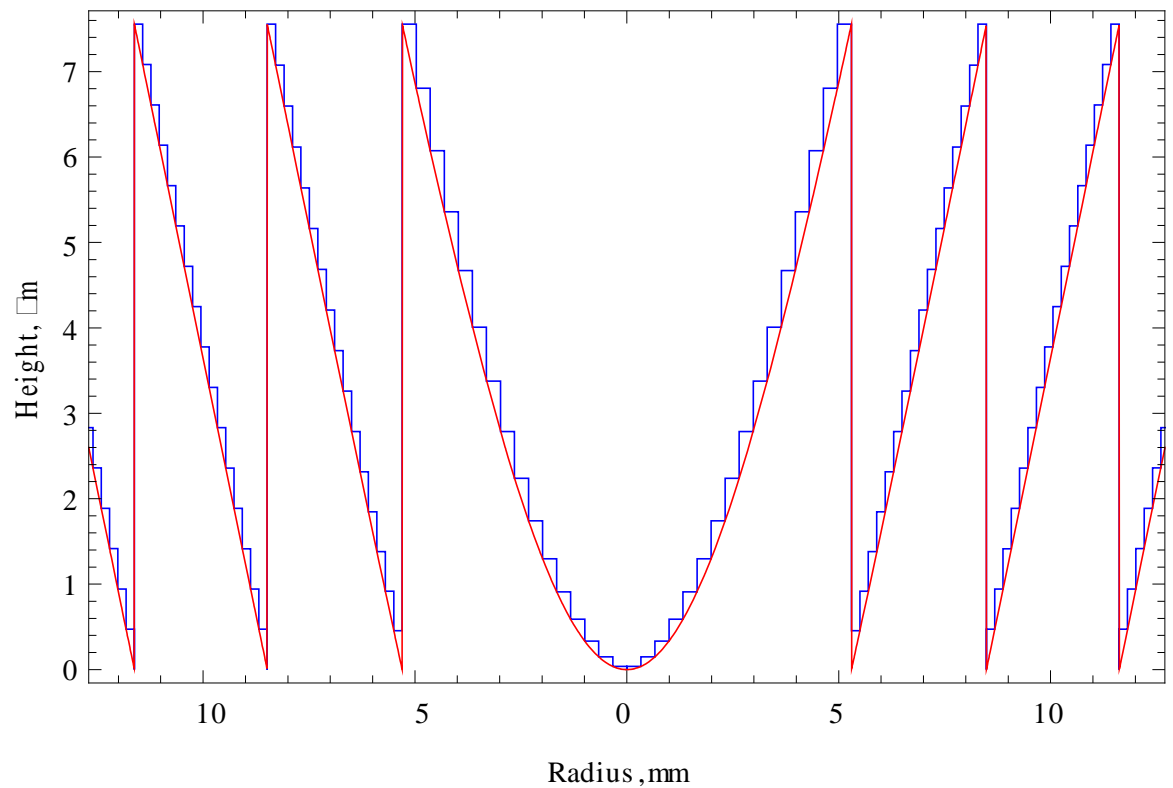

Figure 1 Calculated DOE height profile. It is a 16-level diffractive element made from ZnSe. The profile ranges from 0 to approximately $7.5 \mu \mathrm{m}$, adapted from reference 8 .

\subsection{Design and fabrication of the DOE}

The DOE was designed following the method outlined by F.M Dickey $e t \mathrm{al}^{11}$. It was designed to accept a $7 \mathrm{~mm}$ Gaussian beam with a wavelength of $10.6 \mu \mathrm{m}$ as an input beam. The element was designed in-house and then fabricated by II-VI Inc. from a 1.5" diameter ZnSe flat-flat substrate. It was coated to minimize the reflection losses at wavelength of 10.6 $\mu \mathrm{m}$. The data for the fabrication of the DOE is provided in table 1 in terms of a calculated height profile. It was then tested using a Zygo surface interferometer, and the results are shown in figures 2 and 3. Figure 4 shows a photograph of the fabricated DOE which was used in the beam shaping experiments.

Table 1. Comparison of designed and measured parameters for the DOE.

\begin{tabular}{|l|l|l|}
\hline & \multicolumn{1}{|c|}{ Design } & \multicolumn{1}{c|}{ Measured } \\
\hline DOE Height Profile & $7.56 \mu \mathrm{m}$ & $\sim 8.8 \mu \mathrm{m}$ \\
\hline Zone 1 width & $14.8 \mathrm{~mm}$ & $\begin{array}{l}\text { Could not be measured due to } \\
\text { the range limitation of the Zygo }\end{array}$ \\
\hline Zone 2 width & $4.5 \mathrm{~mm}$ & $4.4 \mathrm{~mm}$ \\
\hline Zone 3 width & $4.4 \mathrm{~mm}$ & $4.2 \mathrm{~mm}$ \\
\hline
\end{tabular}

Using the Zygo data presented in Table 1, one is able to compare the design of the DOE to that of fabricated. The zone spacing determines the function of the element, and the height determines the wavelength for which it is suited. The fabricated element shows zone width deviations from the designed element and these deviations imply an optimal operating wavelength exist (other than that of the design of $10.6 \mu \mathrm{m}$ ), assuming there is a constant refractive index of the element ${ }^{12}$. 


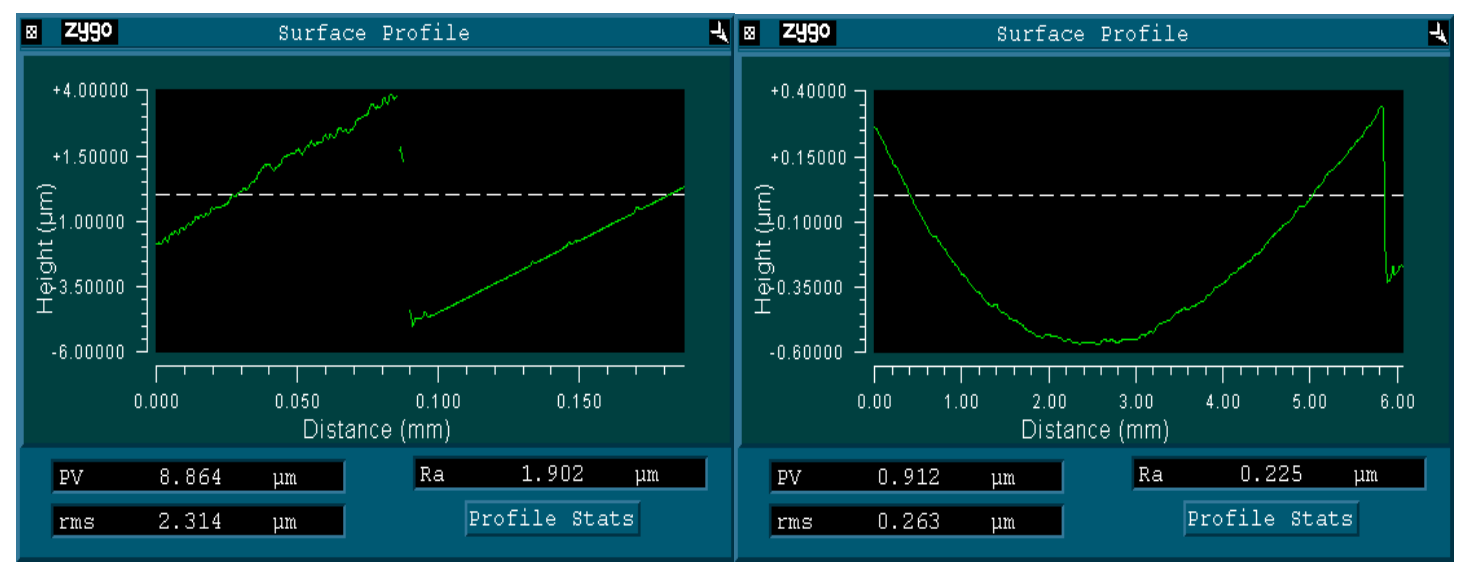

(a)

(b)

Figure 2. Surface profiles across the DOE, with (a) showing a zoomed view of a zone transition and (b) showing the full central region.

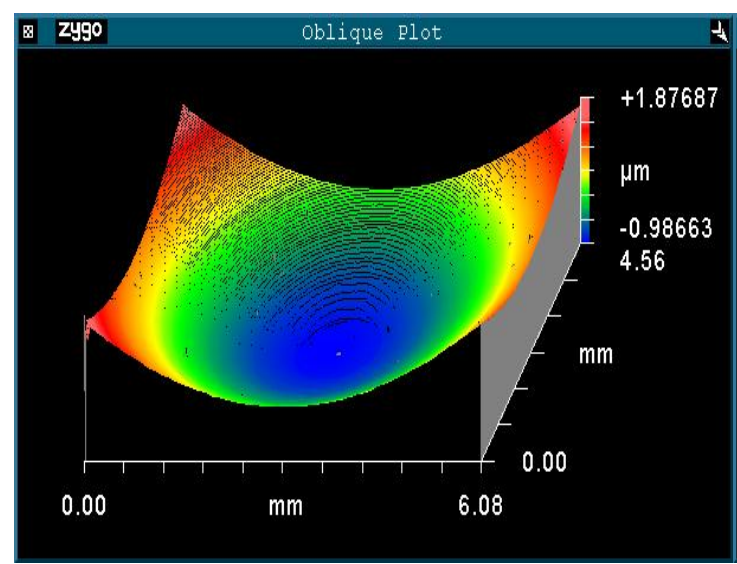

(a)

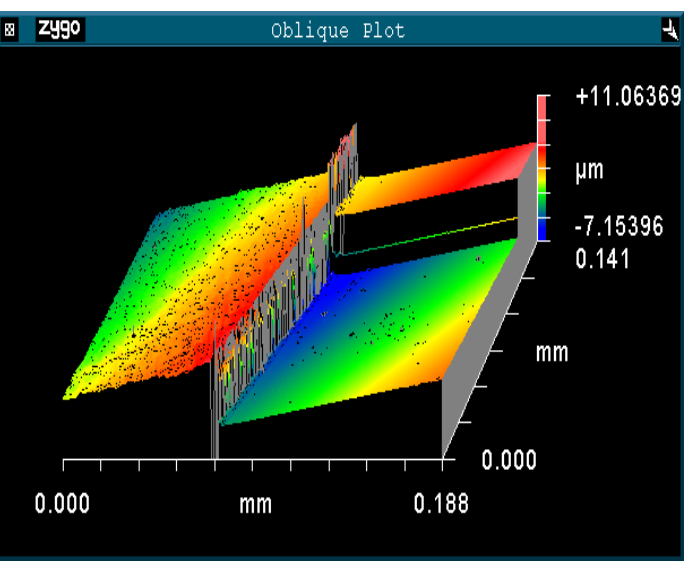

(b)

Figure 3. 3D plots of the surface structure of the DOE, with (a) showing the central region of the element and (b) showing the surface across a zone transition.

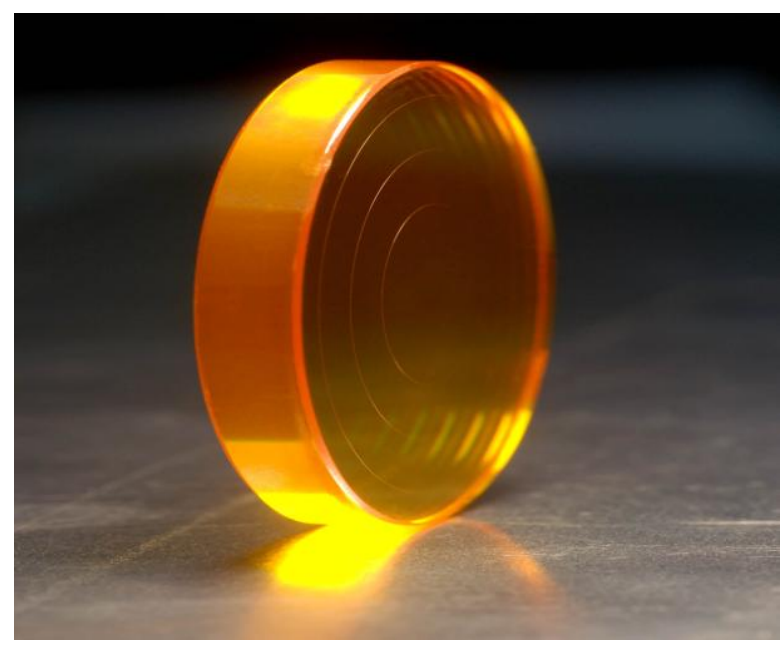

Figure 4. A photograph of the diffractive optical element 


\section{FLATTOP BEAM GENERATION}

This section presents the experimental investigations for generating a flattop beam profile from a Gaussian beam profile using a DOE. The DOE was evaluated using designed and non-designed laser parameters such as wavelength and misalignment effects were also studied. We further investigated the flattop beam propagation behavior. In the sections that follow we discuss the experimental set-up for $\mathrm{CO}_{2}$ laser beam shaping and the experimental results of the performance of the DOE are also presented.

\subsection{Experimental set-up}

Beam shaping experiments were done using a continuous wave (cw) $\mathrm{CO}_{2}$ laser from Edinburgh instruments (Model PL2) as the laser source. The laser is wavelength tuneable $(9 \mu \mathrm{m}$ to $11 \mu \mathrm{m})$, enabling the selection of the desired wavelength by rotating a micrometer dial which is attached to an internal diffraction grating which is used as a back reflector in the laser resonator. Figure 5 shows an experimental set-up for shaping of the $\mathrm{CO}_{2}$ laser.

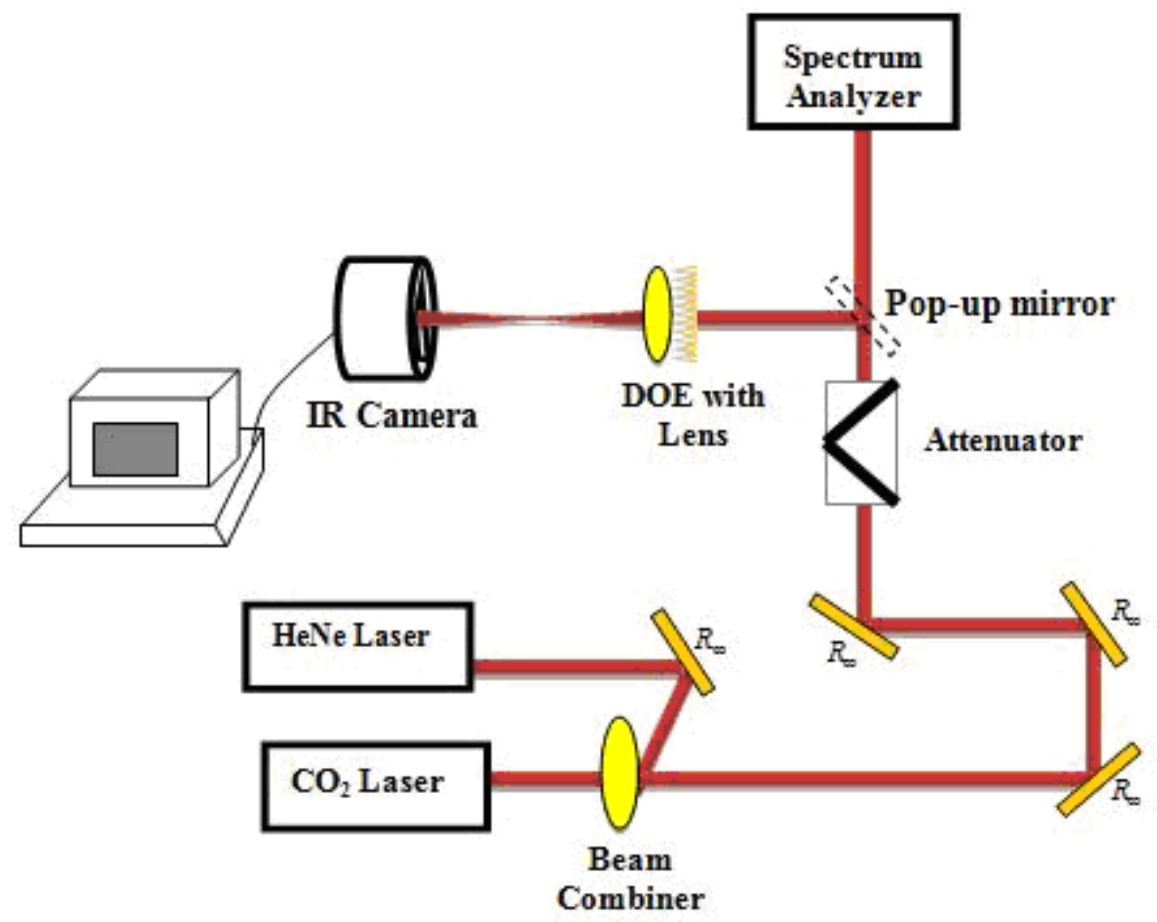

Figure 5. Experimental set-up for $\mathrm{CO}_{2}$ laser beam shaping

An optical engineering spectrum analyzer was used to determine the operating wavelength of the laser, while the attenuator was used to decrease the energy of the laser. The beam shaping optics, which consisted of a DOE in conjunction with a Fourier transforming lens, were placed at the position where a $7 \mathrm{~mm}$ beam radius was located and returned a flattop beam at the propagation distance $z$ from the beam shaping optics.

\subsubsection{Wavelength dependence}

The DOE elements are usually designed to work at a specific wavelength, but theory exists that these element can be made to be wavelength independent ${ }^{8}$ and in this paper, we investigate these theoretical predictions. To investigate the wavelength dependence of the DOE, its performance was tested using the designed wavelength of $10.6 \mu \mathrm{m}$, as well as non-designed wavelengths $(10.2 \mu \mathrm{m}, 9.6 \mu \mathrm{m}$ and $9.3 \mu \mathrm{m})$ in the $\mathrm{CO}_{2}$ laser tuneability range. The results are shown in figure 6. 


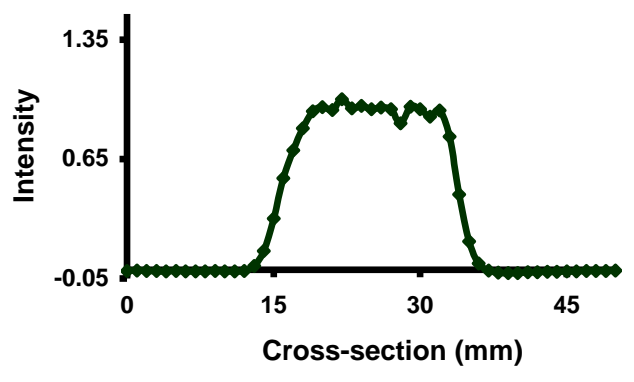

(a)

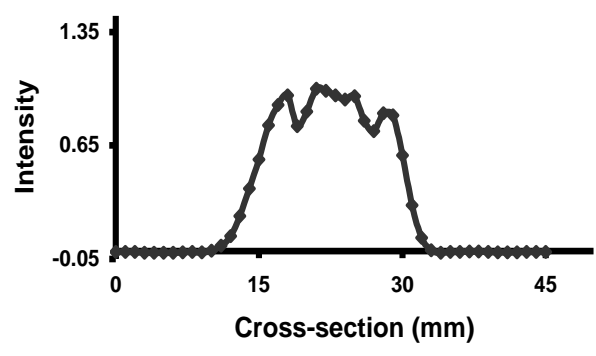

(c)

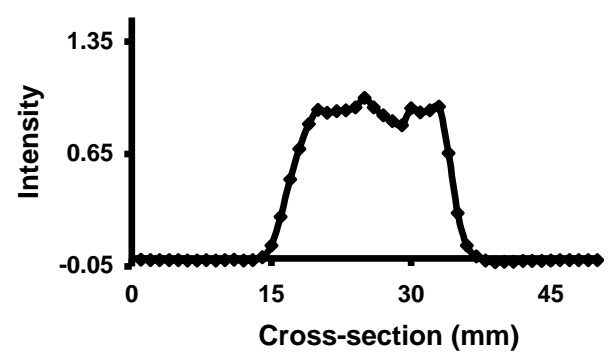

(b)

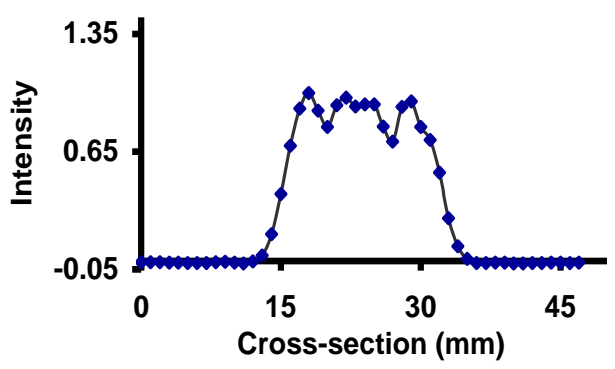

(d)

Figure 6. Output flattop beam at (a) the designed wavelength, $10.6 \mu \mathrm{m}$, and at the three non-designed wavelengths, (b) $10.2 \mu \mathrm{m}$, (c) $9.6 \mu \mathrm{m}$ and (d) $9.3 \mu \mathrm{m}$.

We observed a negligible change in the beam profile induced by the DOE due to a different wavelength. In understanding the reason for this, the wavelength dependence relationship between the DOE height profile and the phase profile needs to be investigated following equation,

$$
\Delta \phi=\frac{2 \pi}{\lambda}<-1 \underline{d}
$$

where $d{ }_{-}$is the DOE height profile, $\Delta \phi$ is the change in phase profile and $n$ is the index of refraction of the DOE. The index of refraction of the material changes slightly for different wavelengths ${ }^{12}$ and it is thus assumed that $n$ is constant in the wavelength region of interest. Thus the effect of changing the wavelength of the input beam was found to be negligible. We conclude that the beam shaping capability of the DOE does not depend on the wavelength of the input $\mathrm{CO}_{2}$ laser, and the advantage of this is that any $\mathrm{CO}_{2}$ laser wavelength may be effectively shaped by the given DOE. 


\subsubsection{Propagation}

The beam profile, after the DOE and Fourier transforming lens, was also investigated at intervals along its propagation. In the curves of the beam profile, given in Figure. 7, the maximum value has been normalized to one.

$340 \mathrm{~mm}$
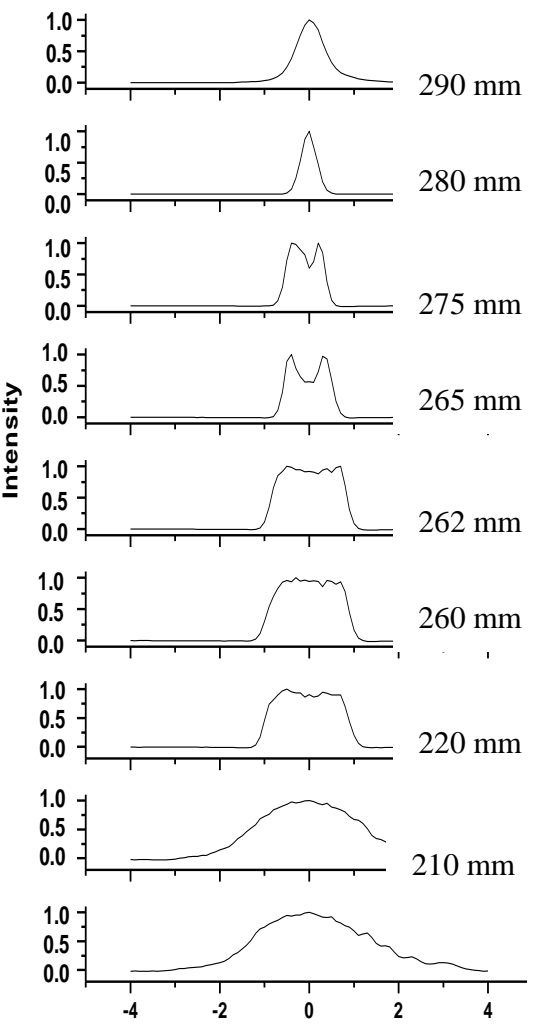

Figure 7. Laser beam propagation at designed wavelength $10.6 \mu \mathrm{m}$. (The number on each curve gives the distance in (mm) from the DOE.

Figure 7 shows the intensity distribution of the propagated profiles at increasing propagation distances from the DOE. We observed that the flattop is generated at a distance between $250 \mathrm{~mm}$ and $265 \mathrm{~mm}$ from the DOE. After the beam propagates more than $265 \mathrm{~mm}$, it rapidly becomes disrupted from uniformity and the beam becomes unrecognizable as a flattop beam. Instead, the beam becomes a Gaussian beam in the far field. Depending on the beam shaping application at hand, this propagation behavior of the flattop beam may be undesirable and the second element may be needed to correct for the phase profile such that the flattop profile may be maintained over a longer propagation distance.

We further observed that the propagation distances at which the flattop beams are generated differ with wavelength. However, the beam shaping system assumes that the input Gaussian beam has a uniform (Flat) phase with a $7 \mathrm{~mm}$ beam radius at the DOE, but it is not always possible to locate a $7 \mathrm{~mm}$ beam radius at the beam shaping element. One possible solution is that the Gaussian beam's phase causes a shift in the location of the output profile ${ }^{11}$. Hence the flattop beam was located a distance from the focal plane of the lens. This was the expected behavior of the performance of the DOE as suggested by the numerical simulations ${ }^{10}$, which show the simulated performance of the DOE, at the design wavelength $(10.6 \mu \mathrm{m})$ and the two non-design wavelengths $(\sim 9.3 \mu \mathrm{m}$ and $\sim 9.6 \mu \mathrm{m})$. 


\subsubsection{Radial off-set}

The effect of a radial offset of the incoming beam on the DOE was also investigated. The output beam profile was investigated when the initial beam was first positioned at the centre of the DOE, and then moved transversely in the -xdirection and $+x$-direction in steps of $0.5 \mathrm{~mm}$ depicted in figure 8 (a) and (b) respectively.

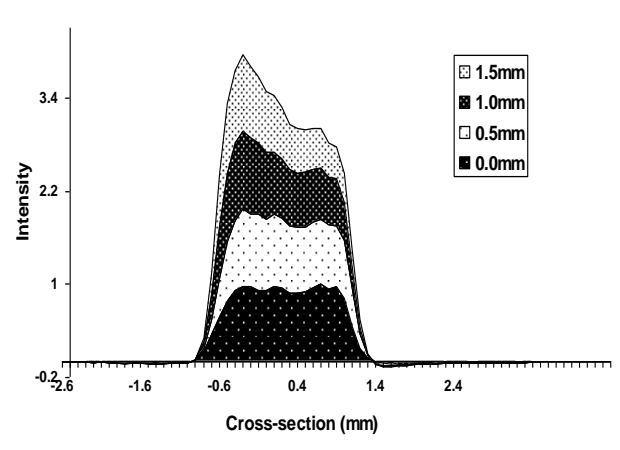

(a)

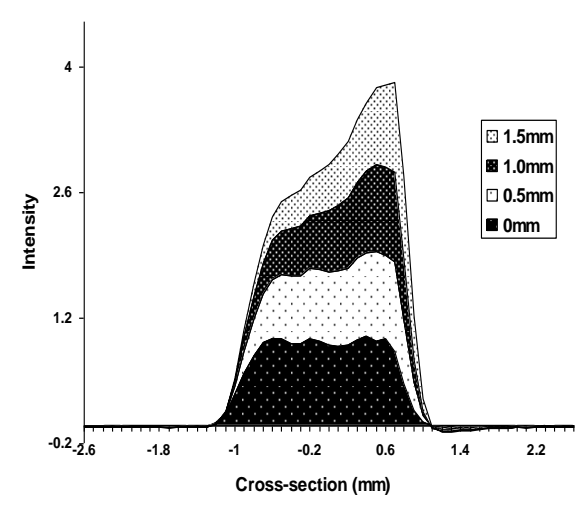

(b)

Figure 8. Representation of the intensity profile due to radial offset of the initial beam on the DOE. The offset step was (a) $\Delta x=-0.5 \mathrm{~mm}$. (b) $\Delta x=+0.5 \mathrm{~mm}$.

Figure 8 shows that a small radial offset of the order of $0.5 \mathrm{~mm}$ of the initial beam on the DOE will easily disrupt the beam shaping ability of the element. It is also apparent that an offset in any particular direction disrupts the intensity profile of the beam in that direction. The intensity distribution deviation is given by the difference between the peak intensity of the disrupted flattop and the peak intensity of the flattop beam when the input beam was positioned at the centre of the DOE. The dependence of the peak intensity deviation was investigated as a function of the offset and the results are presented in figure 9 .

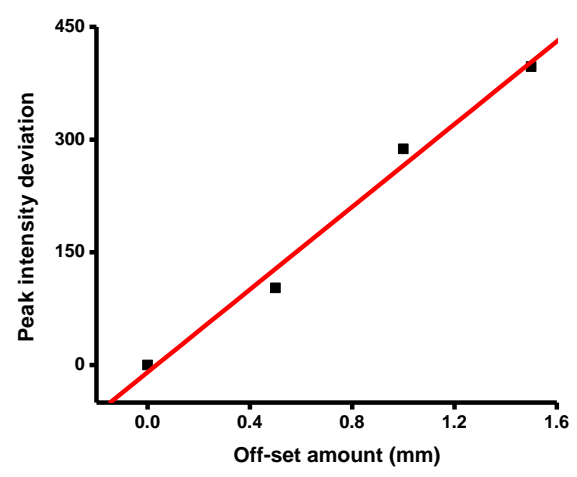

Figure 9 . The intensity peak deviation of the flattop beam by a radial off-set of the initial beam on the DOE as a function of the off-set.

Figure 9 shows a linear dependence of the peak intensity deviation on the offset, with an increase in the offset amount resulting in an increase in the peak intensity deviation of the flattop beam. This suggests that the grater the offset amount of the incident beam on the DOE, the more the output flattop beam is disrupted. The disrupted flattop beam of figure 8(a) and figure 8(b), would not give uniform illumination as intended, and could cause severe distortion in the process for which it is being applied. 


\section{CONCLUSIONS}

A method for converting a Gaussian intensity profile to a flattop intensity profile using a diffractive optical element was described. The performance of the element was tested experimentally to determine its properties under various input conditions. Effects due to the radial offset of the incoming beam on the element have been investigated as well as the effect of varying the input wavelength. It was shown that the element was useful under non-ideal conditions. The DOE was able to convert a Gaussian beam to a flattop beam at four different wavelengths $(9.3 \mu \mathrm{m}, 9.6 \mu \mathrm{m}, 10.2 \mu \mathrm{m}$ and 10.6 $\mu \mathrm{m})$. The effect of wavelength variation on the beam shaping ability of the DOE using several wavelengths of the $\mathrm{CO}_{2}$ laser was found to be negligible and therefore we can conclude that any $\mathrm{CO}_{2}$ laser may be shaped using the DOE and that our DOE is wavelength independent in the range $9 \mu \mathrm{m}-11 \mu \mathrm{m}$. The radial offset of the incident beam on the DOE was found to have a significant impact on the output beam. Thus accurate alignment of the input laser beam onto the DOE is required for good laser beam shaping.

\section{REFERENCES}

[1] Kanzler, K., "Diffractive laser beam shaping for material processing using a $\mathrm{CO}_{2}$ laser," Proc. SPIE, 5525, 64-75, (2004).

[2] Walker, E. and P., Milster, T., D., "Beam shaping for optical data storage,” Proc. SPIE, 4333, 73-92, (2001).

[3] Bashkatov, A., "Optical properties of human skin, subcutaneous and mucous tissues in the wavelength range from 400 to 2000 nm," J. Phys, D: Appl. Phys., 38, 2543-2555, (2005).

[4] Lee, W., "Method for converting a Gaussian laser beam into a uniform beam," Appl. Opt., 22, 3644-3647, (1983).

[5] Shafer, D., "Gaussian to flattop intensity distributing lens," Opt. laser technol., 14, 159-160, (1982).

[6] Hoffnagle, J., A. and Machael, J., C., "Design and performance of a refractive optical system that converts a Gaussian to a flattop beam," Appl. Opt., 39, 5488-5499, (2000).

[7] Taghizadeh, R., "Design and fabrication of diffractive elements for laser material processing applications," Opt. and lasers in eng., 34, 289-307, (2000).

[8] Forbes, A., du Plessis, A., and Rohwer, E., G., "Comparison of laser beam shaping by diffractive and refractive method," Proc. SPIE, 58760H-1, (2005).

[9] Thaning, A., "Asymptotic techniques in design and characterization of diffractive axicon," Thesis for the degree of licentiate of technology, Royal institute of technology, (2002).

[10] Romero, L., A. and Dickey, F., M., "Lossless laser beam shaping," J. Opt. Soc. Am. A, 13, 751-760, (1996).

[11]Dickey, F., M. and Holswade, S., C., "Gaussian laser beam profile shaping." Opt. Eng., 35(11), 3285-3295, (1996).

[12] www.cvilaser.com/Common/PDFs/Index_of_Refraction.pdf. 